\title{
Study on Tactics, Karate Athletes in China
}

\author{
Ren Yadong \\ Huaiyin Normal University, Jiangsu, Huaian223300 \\ hunter2011@foxmail.com
}

Keywords: Karate kumite, Basic, Technique,Tactics, Training.

\begin{abstract}
Like the taekwondo, sanshou and other sport events, karate kumite movement also belongs to the fighting antagonistic item. We summarize the technical and tactical use of the outstanding karate kumite athletes in our country to get the characteristics of the technical and tactical application in the athletes' kumite match in this paper. Then we analyze and study the physical training, summarize the regularity to provide the guidance for the kumite athletes in our country to join in the match. We study the advantages and disadvantages of the kumite athletes in our country, and we put forward the suggestion for the technique and tactics training of the kumite athletes. And we provide reference for the kumite athletes in our country to get the excellent achievements in the match.
\end{abstract}

\section{Introduction}

Because different sport events need different requirements for the athletes. So it needs the athletes to have different technique and tactics, and physical quality. According to the characteristics of karate, the corresponding echnique and tactics and physical training should be carried on. Carrying on the physical training is the foundation. It can improve the athletes' physical quality, and it can also promote the grasp of technique and tactics. So the athletes can maintain the best physical condition in the match so that they can use the corresponding tactics to have a match flexibly and reasonablely. Only the athletes have good psychological quality and physical quality can they improve the trainning result of technique and tactics better. In this way the athletes can adapt to the intense and drastic match tempo to make technique and tactics play its role fully and completely.

\section{The Tactical Analysis of Karate Athletes in Our Country}

When the kumite athletes in our country have a match, the main tactics can be seen as follows.

Direct Attack Tactic. Direct attack tactic is to use a simple technique to attack the opponent's effective parts fast and effectively so as to get the scores. It overpowers the opponent in an imposing manner to cause nervous psychology on the opponent to control the initiative of the match. When the kumite athletes adopt the direct attack tactic, their own technical specialty needs to be fully expressed. By using the most skilled and effective technical attack means to control the process of match. The kumite athletes should be able to grasp the opportunity for combat in the match quickly to give full play to their own specialty technique, that is to say, use the direct attack tactic. They can use the offensive techniques combination quickly to make clear of the scoring goals so as to take simple combination of actions to make an attack or make an defense and counterattack for the opponent. When the opportunity for combat in the match is against the athletes themselves, they should take a defensive situation temporarily. They should wait in the form of retreat and autokinetic means to creat the opportunity constantly so as to wai for the emergence of the opportunity for combat.

Tactic of Contending for First Place. The characteristics of tactic of contending for first place is that launching an attack is earlier than the opponent, and then they strike first to gain the initiative. Once the match starts, the kumite athletes should take the fierce attack technique to attack the opponent continuously. They use all kinds of boxing, kicking technique and other technical combinations to force the opponent to be only able to take defense. So the opponent loses the ability 
of counterattack to get the initiative in the match field. This method can consume the physical energy of the opponent constantly to make the opponent have mistakes so as to get the scores by attack. Using the tactic of contending for first place also has some disadvantages and risks. The tactic of contending for first place has much higher demand on the physical energy. It can consume the physical energy quickly. If the opponent has rich match experience and circles round to delay the opponent constantly, it will consume his own physical energy too much so as to get counterattack from the opponent.

Therefore, probing the level of the opponent is the purpose to use the tactic of contending for first place. After we give the adjustment on the preparation of opponent's technique and tactics, if the opponent is no better than himself, he will attrack fiercely to make the opponent be busy dealing with it so as to seize the opportunity to get scores quickly because of the consumption of the physical energy. If the opponent prepares adequately or have less match experience, he will use the tactic of contending for first place to make the opponent be in a passive position and reduce the ability of judgment.

Counterattack Tactic. Counterattack tactic is the technique to carry on the defensive and adopt the counterattack at the same time. The action for the attack characteristics of kumite match is direct. The change is much less. When the opponent have a violent attack positively, our party dwells on the abstract and avoids real issues, and constantly keeps moving. Making use of the opponent's attack gap takes the counterattack quickly after our party defends the opponent's attack successfully so as to make the opponent be difficult to deal with.

The kumite athletes should be good at using active attack to cover the intension of tactical counterattack. Taking the initiative to attack the opponent, constantly stimulating the opponent, and exposing the defects of the opponent makes the opponent's reaction speed become slow and the action become incoherent, and the psychological pressure get large and tense, and so on. In this way the counterattack condition which is good for themselves is created.

Feint Tactic. When there is a kumite match, the tactic which is most commonly used by the athletes is the feint tactic.

The feint tactic refers to that making use of disguised attack action makes the opponent not judge his own real attack direction. By adopting the feint actions constantly let the opponent has wrong reaction to expose the gap.

When there is a match, the kumite athletes must be good at using feint actions or deceptive movements to make the opponent be deceived. According to the opponent's wrong coping the appropriate technical scores can be taken. Before the match begins, using feint tactic to judge the opponent's reaction, speed, tactics and so on to find the opponent's weakness. When the match is at a stalemate, because of physics, psychology and other influences, the feint action can make the opponent be confused to make him make false judgment. The kumite athletes also can adopt the feint attack tactic to make the opponent make mistakes by creating counterattack conditions so as to get scores with quick counterattack.

Intercepting Tactic. Intercepting tactic refers to when the kumite athletes have a match, they face the opponent' $\mathrm{s}$ attack squarely to pass the .successful defense. When the opponent adopts the attack, our party use the mistakes made by my opponent to destroy the opponent's attack routines. So our party can get the scores by attacking the opponent or by destorying the opponent's attack. When our party use the intercepting tactic, the characteristics of the opponent's technique and tactics need to be further understood. So our party can accurately judge the opponent's attack routines to decrease the risk of using the intercepting tactic.

Marginal Tactic. The marginal tactic refers to making use of the competition rules of kumite match forces the opponent to be away from the match field. The kumite athelets should take the initiative attack means or use the means of opposing the force to achieve the purpose of forcing the opponent to the match line position so as to increase the opponent's risk of being punished. If the opponent uses the marginal tactic our party should circle round with the opponent to damage the adjusting pace so as to force the opponent out of the field. 
Rule Tactic. The rule tactic refers to using the disadvantages of match rules, and using the penalty characteristics of referee carry on the rule tactic. The kumite athetes should adopt the rule tactic to get scores according to the match objectives and the reserved technique and tactics. If our party needs to adopt the rule tactic, it needs the coaches and athletes to have deep study arnd grasp of the summarized experience in actual combat for kumite competition rules in the long-term training and match. The usage of related tactics can be calculated out by the video of 2008 national championship. The statistical data can be shown in table 1 .

Table 1 The statistical table of tactical use number of kumite athletes

\begin{tabular}{|c|c|c|c|c|}
\hline type & $\begin{array}{c}\text { use number of } \\
\text { times }\end{array}$ & $\begin{array}{c}\text { Average } \\
\text { numbers of } \\
\text { times per } \\
\text { match }\end{array}$ & $\begin{array}{l}\text { sucessful } \\
\text { number of } \\
\text { times }\end{array}$ & sucessful rate \\
\hline $\begin{array}{c}\text { Direct Attack } \\
\text { Tactic }\end{array}$ & 406 & 20.3 & 134 & 0.33 \\
\hline $\begin{array}{c}\text { Tactic of } \\
\text { Contending for } \\
\text { First Place }\end{array}$ & 140 & 7 & 10 & 0.07 \\
\hline $\begin{array}{c}\text { Counterattack } \\
\text { Tactic }\end{array}$ & 199 & 9.95 & 52 & 0.26 \\
\hline Feint Tactic & 7 & 0.35 & 2 & 0.22 \\
\hline $\begin{array}{c}\text { Intercepting } \\
\text { Tactic }\end{array}$ & 104 & 5.2 & 31 & 0.3 \\
\hline Marginal Tactic & 62 & 3.1 & 6 & 0.09 \\
\hline Rule Tactic & 12 & 0.6 & 0 & 0 \\
\hline
\end{tabular}

Seen from table 1, direct attack tactic is a kind of tactic which is used most by the kumite athetes. The use number of times is 406 and the success rate is the highest. The use numbers of times of feint tactic and rule tactic are much less. The average use number of times per match is less than once, and the success rate is much lower. The type of using the tactic and number of times are much less. The outcome brought by it is that when there is a match the referee's penalty can't be beneficial to themselves, the emotion and technology of the opponent also can't be suppressed, the match atmosphere and rhythm can't be grasped very well, so the form of direct confrontation can be only taken to compar with the opponent. It is not be good for giving full play to their own advanced technologies.

\section{The Strategic Research on improving the Technique and Tactics of Kumite Athelets in Our Country}

The Physical Requirement of Kumite Match. The physical reserve of kumite athelets determines the competence of technique and tactics in actual performance in the match. The humanbody's main three function systems are glycolysis energy system, the original phosphoric acid energy supplying system and the aerobic energy system. In different stages of the match, three kinds of energy supplying systems play a role of different energy supplying functions to provide the energy for the athletes. The glycolysis energy system is main energy supplying system. The aerobic energy system is the basis of the athletes's physical work to determines that the athletes perform anaerobic capacity. 
The duration of kumite match is very long. The athletes need to have some matches for many times. The intensity of match is very big so that it consumes much more physical energy. If the athletes want to deal with the match better to get the outstanding achievements, they must be energetic and have strong ability of oxygen metabolism. If they have no strong aerobic endurance, in the process of match with high strength and high density the kumite athletes cannot perform the level of technique and tactics to affect the results of the match. Therefore, should strengthen the training of the athletes' cardiopulmonary function to make the athletes have higher aerobic capacity so as to make the athletes have the physical advantages. In this way should ensure that the athletes can have a continuous having matches to play their tactics levels smoothly so as to obtain the excellent results. At the same time, the kumite athletes have very good aerobic capacity which can prevent injury to make the athletes recover to the best state rapidly after the match.

Improving the Level of Technique and Tactics by the Special Physical Training. The kumite sports needs the athletes have somplete physical ability, so special physical training is very necessary. In the process of kumite training should cooperate with the characteristics of the special match and use different kinds of training methods and means to make kumite athletes' technique level get improved and enhance the athletes' bearing ability for sports load to make the athletes' sports quality get developed.

Showing the best technique and tactics level and keeping the physical ability for a long time are the athletes's requirements of endurance quality. It needs the special antagonistic practice for a long time or the antagonistic training beyond the routine. The high-intensity confrontations make the athlete's physical reserve be consumed much quicker. By increasing the intensity of the confrontation, it can greatly improve the athletes' special endurance quality. For the training means fighting one more round can be be taken. The training of fighting one more round can effectively use the training time. And it has significant training effect. The trainings of reactions, actions and movements are three types of speed training. By passing the the opponent's visual information of the change in technique and tactics the athletes' reaction capability has training to improve the reaction speed. According to the characteristics of actions in technique and tactics, using the special actions makes the athletes have the training with the maximum speed to improve the action speed. When there is a match, it needs a wide variety of body movements to cooperate. Having a multilateral displacement constantly needs the athletes to exercise muscle strength and improve the body's flexibility. By the high-intense repetitive training the speed of displacement increases.

The Arrangement of Physical Training. Only the athletes has the good strength quality the athletes have a good spot performance to successfully complete the training task. The training of explosive force is the focus of strength training in kumite movements. And at the same time it also needs to have the trainings of maximum strength and strength endurance load. The athletes should make use of unstable training means to have the training. The athletes should make use of barbells, dumbbells and other related equipments for training reasonablely. The training for the intensity of maximum strength is the basis of strength training. To improve the stability of the athletes' muscles in key parts of the body requires to adopt the unstable training method. Making a training plan of special explosive force is used to improve the technical level of upper and lower limbs. Making use of the traning mehods of wheel war and fighting one more round to increase the athletes' strength endurance.

In the process of training, the kumite athletes can use the training of moving, quick short steps, jumping and running to make their own sensitivity and coordinated ability be improved so as to ensure the continuity of the techniques and actions.

\section{References}

[1] M.J. Tian. The Event-group Training Theory. Peoples Sports Publishing House,1998.

[2] M.J. Tian. Theories of Sport Training. Beijing Sport University Press, 2000.

[3] X.R. Chen. The Science of Sport Tactics. Peoples Sports Publishing House, 20002. 